\title{
Waiting lists in coronary artery bypass graft patients and role of coronary angiography: the Apulian experience
}

This article was published in the following Dove Press journal:

Clinical Audit

13 May 2013

Number of times this article has been viewed

\author{
Marco Matteo Ciccone' \\ Ambrogio Aquilino ${ }^{2}$ \\ Giulia Frasso' \\ Francesca Avolio 2 \\ Barbara Contegiacomo 2 \\ Elisabetta Graps ${ }^{2}$ \\ Francesca Cortese ${ }^{2}$ \\ llaria Dentamaro² \\ Vito Petrarolo ${ }^{2}$ \\ Daniela Salvante ${ }^{2}$ \\ Anna Salvatore ${ }^{2}$ \\ Pasquale Caldarola ${ }^{3}$ \\ Pietro Scicchitano' \\ Francesco Bux ${ }^{2}$ \\ 'Department of Emergency and Organ \\ Transplantation (DETO), University \\ of Bari, ${ }^{2}$ Regional Healthcare Agency \\ of Puglia, ${ }^{3}$ Cardiovascular Diseases \\ Department, San Paolo Hospital, Bari, \\ Italy
}

Correspondence: Marco Matteo Ciccone Cardiovascular Diseases Section,

Department of Emergency and Organ Transplantation (DETO), University of Bari, II Piazza G Cesare, Bari 70I24, Italy Tel +39080547 8791

Fax +39080 5478796

Email marcomatteo.ciccone@uniba.it
Purpose: To evaluate waiting lists during the coronary artery bypass graft (CABG) diagnostictherapeutic pathway of patients, in order to detect the actual waiting time between diagnostic evaluation and surgery, and to explore if coronary angiography provides an option as a starting point of waiting lists for CABG patients.

Methods: We analyzed 496 hospital discharge cards (year 2009) from main cardiac surgery units in Apulia, Italy. Exclusion criteria were emergency patients coming from regions other than Apulia, and CABG associated with cardiac valve surgery.

Results: A total of $97.6 \%$ patients underwent $\mathrm{CABG}$ within 0-30 days of their reservation date, and $81.7 \%$ passed from the first diagnostic step to coronary angiography within 0-30 days. The mean time delay in the diagnostic (ie, the time elapsing from the date of first test performed to detect coronary heart disease and that of coronary angiography) and therapeutic (ie, the time elapsing from the date of waiting-list admission and that of cardiac surgery unit admission) phases was $17.3 \pm 31.306$ and $5.09 \pm 9.375$ days respectively; $27 \%$ and $19.8 \%$ underwent $\mathrm{CABG}$ within the same day or at least the day after reservation day, whilst $47.2 \%$ completed the diagnostic phase on the same day.

Conclusion: The waiting lists for CABG surgery diagnostic-therapeutic phase in Apulia are short.

Keywords: waiting lists, $\mathrm{CABG}$, diagnosis, therapeutic phase

\section{Introduction}

The Italian health care system is facing an ongoing and worrisome issue: waiting lists in clinical practice. Cardiovascular diseases are strongly linked to waitinglists problem. ${ }^{1-4}$ Epidemiological data on acute coronary syndrome (ACS) in Italy revealed an incidence of 227.3 per 100,000 for men (aged 25-84 years) and 97.9 per 100,000 for women (aged 25-84 years), and a prevalence of myocardial infarction of $1.5 \%$ in men and $0.4 \%$ in women. These data suggest that delays in ACS treatment affect a huge number of the population, decreasing life expectancy. Considering that the prevalence of chronic and often asymptomatic cardiovascular diseases could be considered even higher than ACS, the importance of evaluating waiting lists in the health system is clear.

The Italian National Program for Waiting List Management 2010-2012 was a mandatory initiative that obliged regions to reduce at minimum time-to-treatment effectiveness by sending real and practical proposals about this matter to the Italian health minister. Cardiovascular and cardiac surgery waiting lists were part of this national evaluation. At the moment, there is no consensus in Italy on an exact 
prespecified time from which a coronary artery bypass grafting (CABG) waiting list should start.

The aims of this study were: (1) to evaluate waiting lists during the diagnostic-therapeutic pathway of CABG patients, in order to detect the actual waiting time between diagnostic evaluation and surgery; and (2) to explore if coronary angiography provides an option as a starting point of the waiting list for $\mathrm{CABG}$ patients, in relation to its crucial role in the diagnostic phase of coronary artery disease patients and its consequential role in directing (or not) patients towards surgery or percutaneous or medical therapy.

\section{Materials and methods Study population}

We analyzed 956 hospital discharge cards (HDCs) from 2009 of all patients admitted to main cardiac surgery units (CSUs) in the Apulia region, Italy, considering only patients who underwent $\mathrm{CABG}$ after a programmed hospital admittance.

HDCs are special cards aimed at gathering information related to patients discharged from private and/or public health departments in national territory. By means of HDCs and in agreement with privacy laws, one finds specific information about the clinical pathway of a patient, from admission till discharge: his/her personal data, every instrumental examination undertaken and its related dates, the final diagnosis, etc. These HDCs allowed us to collect all information in a relatively simple manner in order to satisfy our study aims.

Exclusion criteria during CSU HDC analysis were: (1) urgent admission to a CSU for CABG; (2) active and passive mobility, ie, patients coming from regions other than Apulia; and (3) CABG associated with cardiac valve surgery, because in these cases $C A B G$ was not the main surgery but was necessary due to valve dysfunction. ${ }^{6}$

The patients underwent CABG surgery in agreement with the current guidelines on myocardial revascularization therapy. ${ }^{6}$ When angioplasty and percutaneous techniques were an alternative to $\mathrm{CABG}$, patients could not be included in our research. In fact, their HDCs did not show any indication of CABG interventions, thus they could not be evaluated by our research team.

We included only patients whose HDCs had a code for $\mathrm{CABG}$ and for cardiovascular diseases, not considering cases without an admission reservation. The first step to detect the start of the diagnostic phase was to focus attention on the diagnostic events before therapeutic admittance of the patients to the CSU. In particular, we took into account diagnostic tests performed up to 180 days before therapeutic hospitalization, using HDC flow and ambulatory assistance codes:

- exercise stress tests

- myocardial perfusion scintigraphy

- echocardiography at rest and during pharmacological/ exercise stress

- cardiac computer tomography

- cardiac magnetic resonance.

In the case of several tests performed within the previous 6 months, we considered the one nearest to the therapeutic admission date. We included cases in which the first diagnostic test date coincided with that of coronary angiography. The date of test execution is important to establish the beginning of the diagnostic phase, because that is the moment in which the specialist confirms the need for coronary angiography evaluation.

Coronary angiography was considered the most recent test to conclude the diagnostic phase before reserving a place in the CSU. Naturally, a patient could undergo coronary angiography several times within the preceding 6 months; we only considered the nearest one to the therapeutic admission.

Coronary angiography has been considered as the leading point from which a waiting list for CSU admission should start. The choice of this test had not been compared to others, and maybe this could be considered a limitation of the present research. Nevertheless, we thought that this is a fundamental test, the latest test in order to judge a patient suitable or not for CABG intervention. At the moment, there is not any test able to distinguish patients as suitable or not for CABG procedures. Furthermore, in agreement with the observational nature of the present work, coronary angiography is easily traceable in the analysis performed, because it was well defined by mean of the specific codes in the HDCs considered. Before coronary angiography, a stable patient cannot be included in a waiting list, because no information can be detected about the morphological and even functional condition of coronary vessels. All these considerations led us to choose the date of coronary angiography as the starting point for a waiting list.

The procedure excluded cases in which it was possible to trace the reservation admission date, but on that date the patients underwent the first diagnostic test and the coronary angiography and surgical procedure was performed: those are clearly cases of urgent events erroneously considered "programmed."

The criteria described refer to the National Program for Waiting List Management: 2010-2012, which provides 
guidelines to monitor waiting lists during CABG diagnostictherapeutic pathways.

According to the aforementioned procedures, the final number of patients involved in our analysis was 518 , although we did not consider 22 patients belonging to the group who underwent urgent surgical revascularization therapy. For this reason, the final number of HDCs evaluated was 496 (51.9\% of all the patients discharged in 2009 that underwent CABG). The present study was approved by ethical committee and is in agreement with the principles of the Declaration of Helsinki. It was also approved by the National Ministry of Health.

\section{Statistical analysis}

Continuous variables were expressed as means and standard deviation, or number and percentages.

\section{Results}

\section{Possible evolution of diagnostic- therapeutic pathway}

Figure 1 resembles a scheme of actual evaluation of patients with coronary artery disease and their natural clinical history and pathway till the eventual CABG procedure, through diagnostic and therapeutic phases of such a process.

In particular, we defined "A" as the date of first test performed during the diagnostic phase; "B" corresponded to the date of the end of diagnostic phase, ie, the date of coronary angiography performance; "C" represented the date when patients were included in a reservation list in the CSU, ie, the beginning of the therapeutic phase; and finally "D" was the date resembling the end of the therapeutic phase, ie, admission to the CSU in order to perform the CABG procedure.

The periods between these dates were used in order to compute the different delays between the phases considered in $\mathrm{CABG}$ clinical pathways of the patients. We found different possible cases in agreement with the delays between phases, and above all between dates:

- Case 1 (A $<>$ B; B $<>$ C; C $<>$ D): this describes a situation in which the four tracing dates were sequentially ordered; 39 patients $(7.9 \%)$ were included in this category.

- Case 2 ( $\mathrm{A}<>\mathrm{B}$; $\mathrm{B}<>\mathrm{C} ; \mathrm{C}=\mathrm{D})$ ): in this case, the first examination date was different from that of the coronary angiography. This was different from the admission reservation date, which was the same as that of admission. Following the diagnostic phase, a reservation was made on another date, and without any delay the patient was admitted to the CSU; 14 patients (2.8\%) were included in this category.

- Case $3(\mathrm{~A}<>\mathrm{B}$; $\mathrm{B}=\mathrm{C}$; $\mathrm{C}<>\mathrm{D})$ : in this case, the first examination date was different from that of coronary angiography, but the same as the reservation date. CSU admission happened some days later; no patients $(0 \%)$ were included in this category.

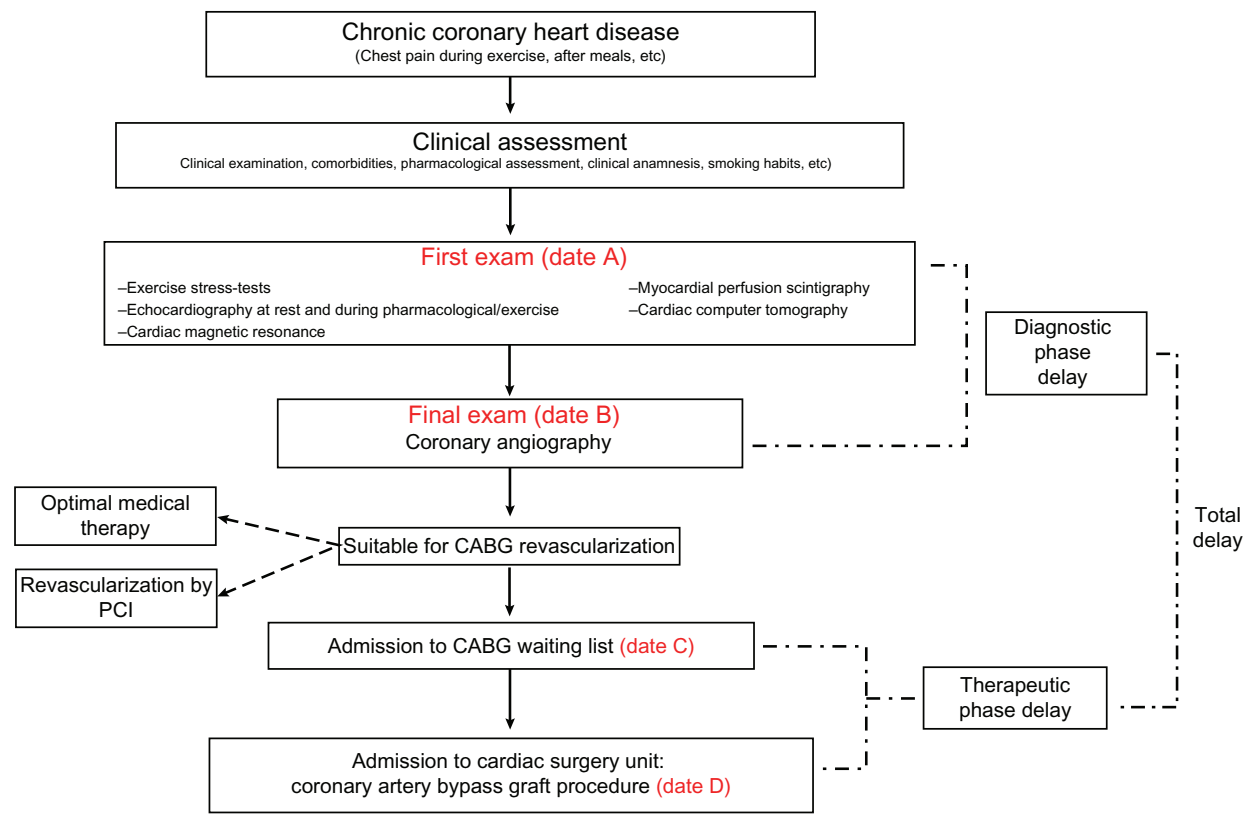

Figure I Coronary heart disease patients' evaluations and clinical pathway till the eventual coronary artery bypass graft (CABG) procedure. Abbreviation: $\mathrm{PCl}$, percutaneous coronary intervention. 
- Case 4 ( $\mathrm{A}<>\mathrm{B}$; $\mathrm{A}<>\mathrm{C}$; $\mathrm{B}=\mathrm{D}$; $\mathrm{B}<>\mathrm{C})$ : in this case, some days after the first examination, admission reservation to CSU was planned. During the hospital stay, coronary angiography and surgery were performed; 127 patients $(25.6 \%)$ were included in this category.

- Case 5 ( $\mathrm{A}<>\mathrm{B} ; \mathrm{B}=\mathrm{C}=\mathrm{D})$ : in this case, after the first diagnostic examination, admission to the CSU was planned. On that very same date, coronary angiography and CABG surgery were performed; 81 patients $(16.3 \%)$ were included in this category.

- Case 6 ( $\mathrm{A}=\mathrm{B}$; $\mathrm{A}<>\mathrm{C}$; $\mathrm{A}<>\mathrm{D}$; $\mathrm{C}<>\mathrm{D})$ ): in this case, all the diagnostic phases were performed at the same time. After this, a reservation for CSU admission was made, which happened some days later; 114 patients $(23 \%)$ were included in this category.

- Case $7(\mathrm{~A}=\mathrm{B} ; \mathrm{B}<>\mathrm{C} ; \mathrm{C}=\mathrm{D})$ : in this case, the diagnostic phase was completed all at once. The admission reservation was made on another date and corresponded with the admission date for CABG surgery; 39 patients $(7.9 \%)$ were included in this category.

- Case $8(\mathrm{~A}=\mathrm{B}=\mathrm{C} ; \mathrm{A}<>\mathrm{D})$ : in this case, the patient underwent first diagnostic evaluation, coronary angiography, and CSU reservation at the same time. CSU admission happened some days later; only two patients $(0.4 \%)$ were included in this category.

- Case $9(\mathrm{~A}=\mathrm{C} ; \mathrm{A}<>\mathrm{B} ; \mathrm{B}=\mathrm{D})$ : in this case, the patient completed the first examination of the diagnostic phase, and once results were in, admission to the CSU was planned. During the hospital stay, the patient underwent coronary angiography and the surgery; only one patient $(0.2 \%)$ was included in this category.

- Case $10(\mathrm{~A}=\mathrm{B}=\mathrm{D}$; $\mathrm{A}<>\mathrm{C}$; $\mathrm{B}<>\mathrm{C}$; $\mathrm{D}<>\mathrm{C})$ : this is a special case. Maybe the patient had been examined and evaluated by a physician. For this reason, the CSU admission had been planned. The patient was admitted to hospital on another date, and during the hospital stay he or she underwent the first real diagnostic examination, coronary angiography, and CABG surgery; 79 patients $(15.9 \%)$ were included in this category.

Finally, we should mention an eleventh case $\mathrm{A}=\mathrm{B}=\mathrm{D}=\mathrm{C}, 22$ patients, in which all the dates coincide. Nevertheless, as previously mentioned, these patients underwent urgent surgical revascularization therapy, and for this reason we could not include them because our analysis only included programmed admissions.

Furthermore, Table 1 shows all the mean time delays for each case considered in every phase evaluated. In particular, it represents mean days for each case during diagnostic and
Table I Time delays: mean days for each case during diagnostic and therapeutic phases and the whole phase

\begin{tabular}{|c|c|c|c|}
\hline & $\begin{array}{l}\text { Diagnostic } \\
\text { phase }\end{array}$ & $\begin{array}{l}\text { Therapeutic } \\
\text { phase }\end{array}$ & Whole phase \\
\hline & $\begin{array}{l}\text { Mean days' } \\
\text { delay }(\mathrm{n})\end{array}$ & $\begin{array}{l}\text { Mean days' } \\
\text { delay }(\mathrm{n})\end{array}$ & $\begin{array}{l}\text { Total mean days' } \\
\text { delay }(n)\end{array}$ \\
\hline Case I & 45 & 13 & 35 \\
\hline Case 2 & 36 & 0 & 38 \\
\hline Case 3 & 0 & 0 & 0 \\
\hline Case 4 & 29 & 6 & 24 \\
\hline Case 5 & 33 & 0 & 25 \\
\hline Case 6 & 0 & 5 & 13 \\
\hline Case 7 & 0 & 0 & 14 \\
\hline Case 8 & 0 & 10 & 10 \\
\hline Case 9 & 5 & 5 & 5 \\
\hline Case 10 & 0 & 7 & 7 \\
\hline Case II & - & - & - \\
\hline
\end{tabular}

Note: Comprising the temporal gap between diagnostic and therapeutic phases.

therapeutic phases and the whole phase (comprising the temporal gap between diagnostic and therapeutic phases).

\section{Time delays in CSU admission: evaluation of single cases}

Table 2 shows that with all the results from each case, there was prompt admission of patients to the CSU. In total, 484 patients $(97.6 \%)$ underwent the final therapeutic phase within 0-30 days of their reservation date, and only one

Table 2 Delays in diagnostic/therapeutic phase reservations

\begin{tabular}{|c|c|c|c|c|}
\hline & 8-30 days & $3 I-90$ days & $\geq 9$ I days & Total \\
\hline \multicolumn{5}{|c|}{ Delay in diagnostic phase, $\mathbf{n}(\%)$} \\
\hline Case I & $18(3.7)$ & $16(3.2)$ & $5(1.0)$ & $39(7.9)$ \\
\hline Case 2 & $9(1.8)$ & $3(0.6)$ & $2(0.4)$ & $14(2.8)$ \\
\hline Case 3 & $0(0)$ & $0(0)$ & $0(0)$ & $0(0)$ \\
\hline Case 4 & $84(16.9)$ & $36(7.3)$ & $7(1.4)$ & $127(25.6)$ \\
\hline Case 5 & $59(11.9)$ & II (2.2) & II (2.2) & 81 (16.3) \\
\hline Case 6 & II $4(23.0)$ & $0(0)$ & $0(0)$ & II 4 (23.0) \\
\hline Case 7 & $39(7.9)$ & $0(0)$ & $0(0)$ & $39(7.9)$ \\
\hline Case 8 & $2(0.4)$ & $0(0)$ & $0(0)$ & $2(0.4)$ \\
\hline Case 9 & I $(0.2)$ & $0(0)$ & $0(0)$ & I $(0.2)$ \\
\hline Case 10 & $79(15.9)$ & $0(0)$ & $0(0)$ & 79 (I5.9) \\
\hline Total & 405 (8I.7) & $66(13.3)$ & $25(5.0)$ & $496(100)$ \\
\hline \multicolumn{5}{|c|}{ Delay in therapeutic phase, $n(\%)$} \\
\hline Case I & $36(7.3)$ & $2(0.4)$ & I $(0.2)$ & $39(7.9)$ \\
\hline Case 2 & $14(2.8)$ & $0(0)$ & $0(0)$ & $14(2.8)$ \\
\hline Case 3 & $0(0)$ & $0(0)$ & $0(0)$ & $0(0)$ \\
\hline Case 4 & $124(25)$ & $3(0.6)$ & $0(0)$ & $127(25.6)$ \\
\hline Case 5 & 81 (16.3) & $0(0)$ & $0(0)$ & $81(16.3)$ \\
\hline Case 6 & III (22.4) & $3(0.6)$ & $0(0)$ & II $4(23.0)$ \\
\hline Case 7 & $39(7.9)$ & $0(0)$ & $0(0)$ & $39(7.9)$ \\
\hline Case 8 & $2(0.4)$ & $0(0)$ & $0(0)$ & $2(0.4)$ \\
\hline Case 9 & I $(0.2)$ & $0(0)$ & $0(0)$ & I $(0.2)$ \\
\hline Case 10 & $76(15.3)$ & $3(0.6)$ & $0(0)$ & 79 (I5.9) \\
\hline Total & $484(97.6)$ & II (2.2) & I (0.2) & $496(100)$ \\
\hline
\end{tabular}


$(0.2 \%)$ exceeded 90 days of his/her reservation date. The diagnostic phase shows that 405 patients $(81.7 \%)$ passed from the first diagnostic step to coronary angiography within 0-30 days. Nevertheless, $25(5.0 \%)$ patients had to wait more than 90 days after the first test to undergo coronary angiography.

The values in Table 3 are expressed in percentage, mean, median, and standard deviation. The minimum and maximum were calculated. Table 3 describes the following:

- The mean time delays in the diagnostic and therapeutic phases were $17.3 \pm 31.306$ and $5.09 \pm 9.375$ days, respectively.

- The medians calculated were 3.5 days and 2 days.

This means that few days elapsed between each phase of the clinical and surgical pathway followed by the patients. And if we consider that $27 \%$ and $19.8 \%$ received their surgery within the same day or at least the day after reservation (data not shown), we could really consider intervention for each patient to be almost immediate. In fact, at least $47.2 \%$ of patients also completed the diagnostic phase on the same day (data not shown). These data show an overall short delay in waiting lists for patients programmed for CABG surgery.

\section{Discussion}

Waiting lists for cardiac surgery are a serious problem in the general economy of the national health-care system. Sobolev et $\mathrm{al}^{2}$ already demonstrated that delays in the admission of patients to a CSU increase in-hospital mortality when considering patients in "semiurgent" (ie, unstable or stable angina, left-main stenosis more than $50 \%$, triple-vessel disease, or double-vessel disease with significant proximal left anterior descending stenosis and impaired left ventricular function') or "nonurgent" conditions (ie, stable symptomatic patients with double-vessel disease with no lesion in the proximal left anterior descending artery and normal left ventricular function, or single-vessel disease with significant stenosis of the proximal left anterior descending artery). ${ }^{6}$ Furthermore, Sampalis et $\mathrm{al}^{7}$ pointed out that waiting lists longer than 3 months for admission to hospital reduce

Table 3 Delay time within diagnostic and therapeutic phases

\begin{tabular}{lll}
\hline & $\begin{array}{l}\text { Diagnostic } \\
\text { phase }\end{array}$ & $\begin{array}{l}\text { Therapeutic } \\
\text { phase }\end{array}$ \\
\hline Number of patients & 496 & 496 \\
Mean (days) & 17.30 & 5.09 \\
Median (days) & 3.50 & 2.00 \\
Standard deviation & 31.306 & 9.375 \\
Minimum (days) & 0 & 0 \\
Maximum (days) & 180 & 114 \\
\hline
\end{tabular}

physical and social skills of patients and make the rate of postoperative adverse events higher than in subjects operated on quickly after diagnosis and any time within 3 months. Thus, a program aiming at reducing waiting lists will further improve the prognosis of patients suffering from severe coronary heart disease. Unfortunately, at the moment we have no data about the real impact on prognosis of our program, and further research will be developed in order to improve results. On the other side, it is quite surprising to note that previous guidelines ${ }^{6}$ do not consider waiting-list problems in their statements. For this reason, this research could add more insight into this complex problem.

If we succeed in further shortening cardiac surgery waiting lists, maybe at best to 1 day from coronary angiography, even in the case of "programmed" admissions to CSU, we will certainly reduce the mortality burden of cardiovascular diseases. The Apulia waiting list was demonstrated to be already short, providing fast admission for stable patients in the cardiac surgery department. Nevertheless, the program has not been able to further reduce the time elapsing between symptoms and diagnosis and between diagnosis and surgical intervention. These times are really important in order to improve the quality of life and treatment of patients. According to us, this would be obtained by potentiating the medical assistance and the health system: further economic efforts should be engaged in in order to consider more physicians and more instruments able to early detect diseases and early provide treatment. The ongoing economic crisis worldwide reduces the probability of rapid implementation, but one should consider that these interventions would not increase economic deficits due to the reduced incidence of coronary heart disease complications.

Furthermore, analysis of our data on time delay in diagnostic and therapeutic phases reveals the high variance in temporal gaps between them. This could account for cases characterized by long waiting in the list of admission to a CSU. We think that this gap could be due to patients' conditions (more "acute" patients will deserve more urgent admission, and this will reduce the possibility of admission of other less urgent patients), to the same waiting-list length, to the few physicians and instrumentation available, etc. Further evaluations are needed in order to assess the real factors able to influence such a gap.

We aimed to establish the role of coronary angiography as the best moment to be considered as starting point of waiting lists for CABG. For this reason, we used HDCs: these are official and traceable documents that show the clinical and surgical pathway followed by the patients and 
are easily identifiable with well-known codes. We thought that coronary angiography is the significant test that makes a surgeon decide whether to operate on a patient or not. Actually, heart surgeons' reports could be considered more appropriate than coronary angiography, but these are difficult to trace. Most reports, in fact, are not registered either in the patients' files or in HDCs. On the other hand, coronary angiography is always registered in HDCs, and therefore was easily evaluated in our research on standard conditions. The absence of the heart surgeons' reports is not relevant as an indication of CABG surgery, because we picked out those cases in which patients underwent coronary angiography, and that test certainly oriented the heart surgeon's choice toward the surgical procedure. Weekly peer-review conferences of cardiovascular specialists could try to decide on advancement or not in waiting lists. ${ }^{8-10}$ However, "priority criteria" can be considered a solution in the case of extremely long waiting lists associated with several urgent needs for revascularization treatments. Due to the short time-to-treatment of stable patients, weekly conferences adopted in order to better take care of CABG patients are useless and not cost-effective.

Coronary angiography has to be considered the leading marker date to program an admission to a CSU. Other studies ${ }^{11}$ tried to validate methodologies to reduce the length of the diagnostic-therapeutic pathway, mainly with the aim of bringing down to zero the number of patients waiting between 31-90 days or even more than 90 days.

Furthermore, Sobolev et al $^{12}$ underlined that waiting-list elongation could depend on urgent admissions, ie, urgent patients have priority over those on the waiting list and so delay the surgical treatment of registered and nonurgent patients. This could be observed in our population: urgent patients (not considered) do not face a waiting list; elective patients could undergo a worsening of their clinical condition, transforming them into urgent cases and so avoiding the waiting list. Nevertheless, the number of these patients is too small (only $2.2 \%$ await $31-90$ days and $0.2 \%>90$ days from CSU reservation to day of admission; see Table 2), although every effort should be made to further reduce such a delay.

\section{Limitations}

This was an observational study, and this limits its results. Although theoretically correct, we had no proof that angiography could be used as a real starting point of waiting lists for $\mathrm{CABG}$ intervention. This could be considered a second limitation of the present research.

\section{Conclusion}

Apulia CABG waiting lists are short because the majority of patients considered suitable underwent the procedure within 8-30 days. Coronary angiography can be considered the theoretical starting point of waiting lists, due to its effectiveness in detection of patients suitable or not for the CABG procedure. Further studies are needed in order to improve the results and to shorten the waiting lists further.

\section{Disclosure}

The authors report no conflicts of interest in this work.

\section{References}

1. Ministry of Health. Stato di Salute e Prestazioni Sanitarie nella Popolazione Anziana: Anno 2000. Ministry of Health, Rome. 2003.

2. Sobolev BG, Fradet G, Hayden R, Kuramoto L, Levy AR, FitzGerald MJ. Delay in admission for elective coronary-artery bypass grafting is associated with increased in-hospital mortality. BMC Health Serv Res. 2008;8:185.

3. Sobolev B, Kuramoto L, Levy A, Hayden R. Unplanned emergency surgery in relation to length of wait lists at registration. Clin Invest Med. 2006;29(4):193-200.

4. Sobolev BG, Levy AR, Kuramoto L, Hayden R, Brophy JM, FitzGerald JM. The risk of death associated with delayed coronary artery bypass surgery. BMC Health Serv Res. 2006;6:85.

5. Perugini E, Maggioni AP, Boccanelli A, Pasquale G. Epidemiology of acute coronary syndromes in Italy. G Ital Cardiol (Rome). 2010;11(10): 718-729. Italian.

6. Task Force on Myocardial Revascularization of the European Society of Cardiology (ESC) and the European Association for Cardio-Thoracic Surgery (EACTS). Guidelines on myocardial revascularization. Eur Heart J. 2010;31(20):2501-2555.

7. Sampalis J, Boukas S, Liberman M, Reid T, Dupuis G. Impact of waiting time on the quality of life of patients awaiting coronary artery bypass grafting. CMAJ. 2001;165(4):429-433.

8. Ray AA, Buth KJ, Sullivan JA, Johnstone DE, Hirsch GM. Waiting for cardiac surgery: results of a risk-stratified queuing process. Circulation. 2001;104(12):I92-I98.

9. Langham S, Soljak M, Keogh B, Gill M, Thorogood M, Normand C. The cardiac waiting game: are patients prioritized on the basis of clinical need? Health Serv Manage Res. 1997;10(4):216-224.

10. Southern DA, Izadneghadar M, Humphries KH, et al. Trends in wait times for cardiac revascularization. Can J Cardiol. 2011;27(2):e21-e27.

11. Seddon M, Broad J, Crengle S, Bramley D, Jackson R, White H. Coronary artery bypass graft surgery in New Zealand's Auckland region: a comparison between the clinical priority assessment criteria score and the actual clinical priority assigned. N Z Med J. 2006; 119(1230):U1881.

12. Sobolev BG, Levy AR, Kuramoto L, Hayden R. Chances of late surgery in relation to length of wait lists. BMC Health Serv Res. 2005;5:63. 
Clinical Audit

\section{Publish your work in this journal}

Clinical Audit is an international, peer-reviewed, open access journal focusing on the processes and outcomes of clinical audit in any area of healthcare. All aspects of patient care are addressed within the journal and practitioners from all disciplines are invited to submit their work. Areas covered include: Publication of audits; How an audit has changed practice;

Practical tips on how to do audits and to avoid pitfalls; How audits have changed patient care; Calls and justifications for new audits. The manuscript management system is completely online and includes a very quick and fair peer-review system, which is all easy to use. Visit http://www.dovepress. com/testimonials.php to read real quotes from published authors. 